\title{
A Generalization of the Method of Averages for Overdetermined Linear Systems
}

\author{
By Ned Anderson
}

\begin{abstract}
Necessary and sufficient conditions are established for an existence and uniqueness result for a generalization of the method of averages for overdetermined linear systems. Some practical schemes are given, and a connection with Galerkin's method is indicated.
\end{abstract}

1. Introduction. In [4], an analysis was made, using the notion of the angle between linear subspaces, of the solution of an overdetermined system of linear equations

$$
F x \approx z, \quad z \in E^{n}, \quad x \in E^{m}, \quad n>m,
$$

$F$ an $n \times m$ matrix, by the method of averages. In this method (we use the notation of [4]), the overdetermined system (1) is replaced by the system

$$
G^{T} F y=G^{T} z
$$

where $G^{T}$ is an $m \times n$ matrix of the form

$$
G^{T}=\left[\begin{array}{llllllllllllll}
1 & 1 & \cdots & 1 & 0 & 0 & \cdots & 0 & \cdots & \cdots & 0 & 0 & \cdots & 0 \\
0 & 0 & \cdots & 0 & 1 & 1 & \cdots & 1 & \cdots & \cdots & 0 & 0 & \cdots & 0 \\
\vdots & & & & & & & & & & \\
\vdots & & & & & & & & & & \\
0 & 0 & \cdots & 0 & \cdots & \cdots & 0 & \cdots & \cdots & 1 & 1 & \cdots & 1
\end{array}\right] .
$$

In [4], the matrix $G$ is called a summation matrix, and it is stated there that $G$ "is supposed to be chosen such that $G^{T} F$ is nonsingular".

In the next section, we define a class of matrices, which we call rectangular smoothing matrices, which includes matrices such as $G^{T}$ above as a special case. We show that the condition that $G$ be a rectangular smoothing matrix is necessary and sufficient for $G^{T} F$ to be nonsingular, for all $F$ derived from a Chebyshev system in the manner described below. Thus, we focus on the case when the overdetermined sys-

Received August 29, 1973.

AMS (MOS) subject classifications (1970). Primary 65F20, 65D10.

Copyright $\odot 1975$, American Mathematical Society 
tem is derived from the approximation of a function by a linear combination of members of a Chebyshev system.

2. A Generalized Method of Averages. Let $\varphi_{0}, \varphi_{1}, \cdots, \varphi_{m-1}$ be a Chebyshev system (we use the definition given in, e.g., $[5$, p. 1]) on the interval $[a, b]$, and let approximate values $\left\{z_{i}\right\}_{i=0}^{n-1}$ of a function be given at the abscissae $a=x_{0}<x_{1}<\cdots$ $<x_{n-1}=b,(n>m)$. Let $F$ be the matrix whose $j$ th column is $\varphi_{j}\left(x_{i}\right)$. We shall consider the following class of matrices $G$ :

Definition. A rectangular smoothing matrix $G$ is an $n \times m$ matrix $(n \geqslant m)$ which satisfies

(i) every $m \times m$ minor formed by deleting $n-m$ columns of $G^{T}$ is nonnegative,

(ii) $\operatorname{rank}\left(G^{T}\right)=m$.

Remark. There are $\left(\begin{array}{l}n \\ m\end{array}\right) m \times m$ minors of $G^{T}$ which can be formed in the above way (i.e., with lexicographical order of the columns preserved). Condition (ii) (together with (i)) implies that at least one of these minors is strictly positive.

One can think of the elements of $G^{T} z$ (the right-hand side of (2)) as weighted sums of the approximate values $z_{i}$. Thus, a normalization condition such as

$$
\sum_{j=0}^{n-1} G_{i j}^{T}=1, \quad i=0, \cdots, m-1,
$$

is perhaps natural for applications but not necessary for the result below.

Two examples of such rectangular smoothing matrices are (we give the transpose, $\left.G^{T}\right)$

$$
\left[\begin{array}{ccccc}
1 / 2 & 1 / 2 & 0 & 0 & 0 \\
0 & 1 / 4 & 1 / 2 & 1 / 4 & 0 \\
0 & 0 & 0 & 1 / 2 & 1 / 2
\end{array}\right] \text { and }\left[\begin{array}{cccc}
1 / 2 & 1 / 2 & 0 & 0 \\
0 & 0 & 2 / 3 & 1 / 3 \\
0 & 0 & 1 / 3 & 2 / 3
\end{array}\right] \text {. }
$$

Two matrices which are not rectangular smoothing matrices are

$$
\left[\begin{array}{cccc}
1 / 2 & 1 / 2 & 0 & 0 \\
0 & 0 & 1 & 0 \\
0 & 0 & 1 & 0
\end{array}\right] \text { and }\left[\begin{array}{cccc}
1 / 2 & 1 / 2 & 0 & 0 \\
1 / 4 & 1 / 4 & 1 / 4 & 1 / 4 \\
0 & 0 & 1 / 2 & 1 / 2
\end{array}\right] \text {. }
$$

We note that the rows of $G^{T}$ are linearly dependent in the above two examples, contradicting (ii). A more subtle example, where the rows of $G^{T}$ are linearly independent, is

$$
G^{T}(\alpha)=\left[\begin{array}{cccr}
1 & 0 & 0 & 0 \\
0 & \alpha & 1-\alpha & 0 \\
0 & 1-\alpha & \alpha / 2 & \alpha / 2
\end{array}\right]
$$


The set of values for which $G^{T}(\alpha)$ is a rectangular smoothing matrix is $2-\sqrt{2} \leqslant \alpha$ $\leqslant 1$.

As the reader can verify, for $x_{0}<x_{1}<x_{2}<x_{3}$, and $\varphi_{0}=1, \varphi_{1}=x, \varphi_{2}=x^{2}$; for $\alpha=0$ the determinant of $G^{T} F$ is (for simplicity, we denote the determinant of a matrix $M, \operatorname{det}(M)$, by $|M|)$

$$
\left|\begin{array}{lll}
1 & x_{0} & x_{0}^{2} \\
1 & x_{2} & x_{2}^{2} \\
1 & x_{1} & x_{1}^{2}
\end{array}\right|<0
$$

and for $\alpha=1, \operatorname{det}\left(G^{T} F\right)$ is

$$
\left|\begin{array}{ccc}
1 & x_{0} & x_{0}^{2} \\
1 & x_{1} & x_{1}^{2} \\
1 & \left(\frac{x_{2}}{2}+\frac{x_{3}}{2}\right) & \left(\frac{x_{2}^{2}}{2}+\frac{x_{3}^{2}}{2}\right)
\end{array}\right|>0 .
$$

Thus, for a suitable choice of $\alpha, G^{T} F$ is singular. For $G^{T}(\alpha)$ above, with $2-\sqrt{2} \leqslant$ $\alpha \leqslant 1$, we also have $\operatorname{det}\left(G^{T} F\right)>0$ for every $F$ derived from a Chebyshev system as will follow from the theorem below.

Theorem 1. Let $a=x_{0}<x_{1}<\cdots<x_{n-1}=b$ be a partition $P$ of $[a, b]$. Let $G^{T}$ be an $m \times n$ matrix whose elements may depend on $P$ (but are bounded). Then a necessary and sufficient condition for $\operatorname{det}\left(G^{T} F\right)$ to be positive, for every $F$ derived from a Chebyshev system $F=\left\{\varphi_{0}, \cdots, \varphi_{m-1}\right\}$ on $[a, b]$, is that $G$ be a rectangular smoothing matrix (termed RSM hereafter).

Proof. Sufficiency. Suppose $G$ is a RSM. By the Cauchy-Binet theorem (see [1], or [6, p. 63]), $\operatorname{det}\left(G^{T} F\right)$ can be written as

$$
\operatorname{det}\left(G^{T} F\right)=\sum_{j=1}^{K} g_{j} f_{j},
$$

where $K=\left(\begin{array}{c}n \\ m\end{array}\right)$, and the $g_{j}$ are the $m \times m$ minors of $G^{T}$ whose columns (considered as columns of $G^{T}$ ) are in increasing order. The $f_{j}$ are $m \times m$ minors formed from the rows of $F$, taken with row indices the same as, and in the same order as, the column indices in $g_{j}$.

Since $F$ is an (arbitrary) Chebyshev system and the row indices in $f_{j}$ appear in increasing order, $f_{j}>0$ for all $j$. Also, $g_{j} \geqslant 0$ for all $j$ by the definition of RSM, and at least one of the $g_{j}$ is positive by the remark following the definition of RSM. Thus, $\operatorname{det}\left(G^{T} F\right)>0$.

Necessity. Suppose $\operatorname{det}\left(G^{T} F\right)>0$. Again we use expansion (3). For any Chebyshev system $F$, we have $f_{j}>0$ for all $j$. Since $\operatorname{det}\left(G^{T} F\right)>0$, then at least one of the 
$g_{j}$ must be positive, using (3). Thus, $\operatorname{rank}\left(G^{T}\right) \geqslant m . \operatorname{But} \operatorname{rank}\left(G^{T}\right) \leqslant \min (m, n)=m$. So $\operatorname{rank}\left(G^{T}\right)=m$. Now, suppose that some $m \times m$ minor $g_{r}$ of $G^{T}$ satisfies $g_{r}<0$. If $m=n$, then $g_{r}<0$ is a direct contradiction of $\operatorname{det}\left(G^{T} F\right)>0$. It remains to consider the case $n>m$. The term $f_{r}$ corresponding to $g_{r}$ in expansion (3) is of the form

$$
\left|\begin{array}{cccc}
\varphi_{0}\left(x_{i_{0}}\right) & \varphi_{1}\left(x_{i_{0}}\right) & \cdots & \varphi_{m-1}\left(x_{i_{0}}\right) \\
\varphi_{0}\left(x_{i_{1}}\right) & \varphi_{1}\left(x_{i_{1}}\right) & \cdots & \varphi_{m-1}\left(x_{i_{1}}\right) \\
\cdots & & \\
\varphi_{0}\left(x_{i_{m-1}}\right) & \varphi_{1}\left(x_{i_{m-1}}\right) \cdots \varphi_{m-1}\left(x_{i_{m-1}}\right)
\end{array}\right|
$$

where $i_{0}<i_{1}<\cdots<i_{m-1}$. Let $I_{r}=\left\{i_{0}, i_{1}, \cdots, i_{m-1}\right\}$ and construct a new Chebyshev system $\widetilde{F}$ as follows:

Let $w$ be a positive, continuous function defined on $[a, b]$ satisfying:

$$
\begin{array}{ll}
w\left(x_{l}\right)=1, & l \in I_{r}, \\
w\left(x_{l}\right)=\epsilon, & l \in\{0,1, \cdots, n-1\}-I_{r},
\end{array}
$$

where $\epsilon>0$ will be chosen later.

We define a new Chebyshev system (see $[5$, p. 10]) $\widetilde{F}$ as

$$
\widetilde{F}=\left\{w(x) \varphi_{0}(x), w(x) \varphi_{1}(x), \cdots, w(x) \varphi_{m-1}(x)\right\},
$$

and denote the associated minors by $\widetilde{f}_{j}$. Clearly, $\widetilde{f}_{r}=f_{r}$, while the determinants $\widetilde{f}_{j}$, for $j \neq r$, can be made as small in magnitude as we wish, by choosing $\epsilon$ small enough, since they all contain a factor $\epsilon$ in at least one row. Choosing $\epsilon$ so small that

$$
\left|\sum_{j \neq r} g_{j} \widetilde{f}_{j}\right|<\left|g_{r} \widetilde{f}_{r}\right|
$$

we get $\operatorname{det}\left(G^{T} \widetilde{F}\right)<0$, a contradiction, and the proof is complete.

Remark 1. Theorem 1 is also true for extended Chebyshev systems [5, p. 6], i.e., it holds for generalized Hermite data $z_{i}^{(k)}, k=0,1, \cdots, k_{i}$. One need only take the function $w$ in the proof to be sufficiently differentiable and to satisfy the additional conditions:

$$
w^{\prime}\left(x_{l}\right)=w^{\prime \prime}\left(x_{l}\right)=\cdots=w^{\left(\max k_{i}\right)}\left(x_{l}\right)=0 \quad \text { for } l \in\{0,1, \cdots, n-1\}-I_{r} .
$$

Applying Leibniz' rule to $w \varphi_{i}$, it is clear that the magnitude of $\widetilde{f}_{j}$, for $j \neq r$, can be made as small as we wish by choosing $\epsilon$ small enough.

Remark 2. The definition of RSM above has the following interpretation. We can associate a weak Chebyshev (WT) system [5, p. 3] of $m$ functions, $\Psi=\left\{\psi_{i}\right\}_{i=0}^{m-1}$, with $G$. The $i$ th column of $G$ is the table vector of the function $\psi_{i}(x)$ at the points 
$x_{0}, x_{1}, \cdots, x_{n-1}$. We shall usually think of $G$ as being derived from a particular choice of $\Psi$, and we call $\Psi$ the associated WT-system. We have:

ThEOREM 2. Let $\Psi=\left\{\psi_{i}\right\}_{i=0}^{m-1}$ be a weak Chebyshev system of functions on $[a, b]$. Let $a \leqslant x_{0}<\cdots<x_{n-1} \leqslant b$, with $n \geqslant m$ and suppose that, for at least one subset $\left\{\tilde{x}_{j}\right\}_{j=0}^{m-1}$ of $\left\{x_{l}\right\}_{l=0}^{n-1}$ with $\tilde{x}_{0}<\tilde{x}_{1}<\cdots<\tilde{x}_{m-1}$, we have $\left|\psi_{i}\left(\tilde{x}_{j}\right)\right|>0$. Then the $n \times m$ matrix $G$ with $G_{i j}^{T}=\psi_{i}\left(x_{j}\right)$ is a RSM.

Proof. This follows directly from the definition of a WT system [5, p. 3] and Theorem 1.

Example. Generalized spline functions. See [5, Chapter XI, Lemma 9.2] for necessary and sufficient conditions which a set $\left\{\tilde{x}_{j}\right\}$ must satisfy with respect to the nodes of the splines in order to have $\left|\psi_{i}\left(\widetilde{x}_{j}\right)\right|>0$. The next few sections will be concerned with some RSM's of obvious practical interest.

\section{RSM's with Positive Entries.}

THEOREM 3. Let $\widetilde{G}^{T}$ be an $m \times n$ matrix $(n \geqslant m)$ of rank $m$. For $0 \leqslant i \leqslant m-1$, let $\tau_{i}$ denote the column index of the first nonzero element in row $i$ and let $\nu_{i}$ denote the column index of the last nonzero element in row $i\left(\tau_{i}\right.$ and $\nu_{i}$ are well defined since $\operatorname{rank}\left(\widetilde{G}^{T}\right)=m$, and clearly $\left.\tau_{i} \leqslant \nu_{i}\right)$. Suppose $\widetilde{G}^{T}$, in addition to being of rank $m$, also satisfies:

$$
\begin{aligned}
& \widetilde{G}_{i j}^{T} \geqslant 0, \quad i=0,1, \cdots, m-1 ; j=0,1, \cdots, n-1 \\
& \tau_{i+1} \geqslant \nu_{i}, \quad i=0,1, \cdots, m-2 .
\end{aligned}
$$

Then $\widetilde{G}$ is a RSM.

Proof. The result will follow from the series of lemmas below. To avoid repetition, we shall omit specifying the range of various indices when this is clear from the context.

LEMMA 1. $\tau_{i+1} \geqslant \tau_{i}$. This is obvious.

LEMmA 2. The nonzero elements in any column of $\widetilde{G}^{T}$ appear consecutively.

Proof. Suppose that column $p$ of $\widetilde{G}^{T}$ has at least two nonzero elements (otherwise the assertion of the lemma holds trivially), and suppose that there are two nonzero elements which do not appear consecutively, i.e.,

$$
\widetilde{G}_{i, p}^{T} \neq 0, \quad \widetilde{G}_{i+1, p}^{T}=0, \cdots, \widetilde{G}_{i+k-1, p}^{T}=0, \quad \widetilde{G}_{i+k, p}^{T} \neq 0,
$$

where $k \geqslant 2$. Since $\widetilde{G}_{i, p}^{T} \neq 0$, we have $p \leqslant v_{i}$. This implies $\tau_{i+1} \geqslant p$ by (b). $\widetilde{G}_{i+k, p}^{T} \neq$ 0 implies $\tau_{i+k} \leqslant p$. Hence $\tau_{i+k} \leqslant \tau_{i+1}$. Using Lemma 1 , we get $\tau_{i+k}=\tau_{i+1}$. But $\tau_{i+1} \leqslant p$ since $\tau_{i+k} \leqslant p$, so $\tau_{i+1}=p$. This contradicts $\widetilde{G}_{i+1, p}^{T}=0$.

LEMMA 3. There are at most two nonzero elements in any column of $\widetilde{G}^{T}$.

Proof. Suppose there are three (or more) nonzero elements in the $p$ th column. They must appear consecutively, by Lemma 2 . Suppose that $\widetilde{G}_{i, p}^{T}, \widetilde{G}_{i+1, p}^{T}$, and $\widetilde{G}_{i+2, p}^{T}$ are all nonzero. By an argument similar to that of Lemma $2, \tau_{i}=\tau_{i+1}=p=$ $\nu_{i}=\nu_{i+1}$. This contradicts $\operatorname{rank}\left(\widetilde{G}^{T}\right)=m$. 
LEMMA 4. Let $M$ be a nonzero minor formed from columns $k_{0}, k_{1}, \cdots, k_{m-1}$ of $\widetilde{G}^{T}\left(k_{0}<k_{1}<\cdots<k_{m-1}\right)$. Then

$$
\operatorname{det}(M)=\prod_{i=0}^{m-1} M_{i i}
$$

Proof. The individual terms of $\operatorname{det}(M)$ are of the form

$$
\operatorname{sign}\left\{\begin{array}{llll}
0 & 1 & \cdots & m-1 \\
i_{0} i_{1} & \cdots & i_{m-1}
\end{array}\right\} \cdot M_{0 i_{0}} M_{1 i_{1}} \cdots M_{m-1, i_{m-1}}
$$

where $i_{0}, i_{1}, \cdots, i_{m-1}$ is some permutation of $0, \cdots, m-1$. Consider the $m !-1$ terms which contain at least one inversion (only $M_{00} M_{11} \cdots M_{m-1, m-1}$ has no inversion). Assume for definiteness that $i_{r}>i_{r+1}$ for a certain $r \in\{0,1, \cdots, m-2\}$. Now

$$
M_{r, i}=\widetilde{G}_{r, j}^{T} \text { and } M_{r+1, i_{r+1}}=\widetilde{G}_{r+1, j^{\prime}}^{T}
$$

with $j>j^{\prime}$ since $i_{r}>i_{r+1}$. Suppose $\widetilde{G}_{r, j}^{T} \neq 0$. Since $\tau_{r+1} \geqslant \nu_{r}$ and $\nu_{r} \geqslant j>j^{\prime}$, then $\tau_{r+1}>j^{\prime}$; that is, $\widetilde{G}_{r+1, j^{\prime}}^{T}=0$. Hence, the only possible nonzero term in $\operatorname{det}(M)$ is $\Pi_{i=0}^{m-1} M_{i i}$. $\operatorname{Det}(M) \neq 0$ by assumption, and since $M_{i i}$ are elements of $\widetilde{G}^{T}$, we have $M_{i i} \geqslant 0$. Hence

$$
\operatorname{det}(M)=\prod_{i=0}^{m-1} M_{i i}>0
$$

COROLlaRY. The nonzero $m \times m$ minors of $\widetilde{G}^{T}(m \geqslant 2)$ with $k_{0}<k_{1}<\cdots$ $<k_{m-1}$ are tridiagonal matrices, with $M_{i, i+1} \cdot M_{i+1, i}=0$, and $M_{i i}>0$.

(Proof. Use Lemma 3 and Lemma 4.)

Lemma 4 concludes the proof of Theorem 3.

4. A Method of Local Moments. We consider the following class of matrices whose use as RSM's we call the method of local moments. Suppose $G^{T}$ is an $m \times n$ matrix, $m \leqslant n$, composed of $m_{i} \times n_{i}$ blocks $G_{i}^{T}, i=0,1, \cdots, r-1$, in a way which is clear from the figure below for $r=3$. We require $m_{i} \leqslant n_{i}$ since this is necessary for $\operatorname{rank}\left(G^{T}\right)=m$.

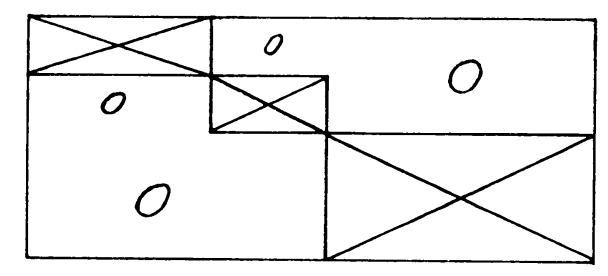

Thus, we require $G^{T}$ to be "block diagonal" with rectangular blocks on the diagonal.

Then we have: 
THEOREM 4. Suppose that for each of the $m_{i} \times n_{i}$ blocks $G_{i}^{T}, i=0, \cdots, r-1$, $G_{i}$ is a RSM. Then $G$ is a RSM.

Proof. This follows by induction on the number of blocks and application of the Laplace expansion [1, pp. 78-82] to the successive nonzero minors with column indices in increasing order. (Only $m_{i} \times m_{i}$ minors of $G_{i}^{T}$ arise as possible nonzero factors in the Laplace expansion of an $m \times m$ minor of $G^{T}$. The sign associated with them is +1 , and each $G_{i}^{T}$ has at least one positive $m_{i} \times m_{i}$ minor.)

Examples.

$$
\left[\begin{array}{ccccc}
1 & 1 & 1 & 0 & 0 \\
e^{x_{0}} & e^{x_{1}} & e^{x_{2}} & 0 & 0 \\
0 & 0 & 0 & 1 & 1 \\
0 & 0 & 0 & x_{3} & x_{4}
\end{array}\right]\left[\begin{array}{ccccc}
1 & 1 & 1 & 0 & 0 \\
x_{0}-x_{1} & 0 & x_{2}-x_{1} & 0 & 0 \\
\left(x_{0}-x_{1}\right)^{2} & 0 & \left(x_{2}-x_{1}\right)^{2} & 0 & 0 \\
0 & 0 & 0 & 1 & 1
\end{array}\right] .
$$

The method of local moments should be especially useful in real-time data processing or in other applications where local "compression" of the equations is warranted. Only a small portion of the data need be in storage at any one time, and the information in a segment of the data is then "compressed" by computing the local moments (the product of $G_{i}^{T}$ and the $i$ th segment of the data vector).

5. A Connection with Galerkin's Method. The method for approximately solving overdetermined systems presented in the previous sections can be seen as a generalization of Galerkin's method [3, Chapter 8.6.4]. Suppose we are seeking an approximate solution

$$
u \approx c_{0} \varphi_{0}+c_{1} \varphi_{1}+\cdots+c_{m-1} \varphi_{m-1}
$$

to a linear operator equation (for a function of one variable) $A u=f$. Galerkin's method requires that the coefficients $c_{i}$ be determined by requiring that the scalar product

$$
\left(\varphi_{i}, A u-f\right)=0, \quad i=0, \cdots, m-1 .
$$

The method we have presented determines the $c_{i}$ by requiring, for a discrete inner product on a net $\left\{x_{j}\right\}_{j=0}^{n-1},\left(\psi_{i}, A u-f\right)=0$, where the $\psi_{i}$ are chosen so that $\psi_{i}\left(x_{j}\right)$ is a RSM. Hopefully, the use of the appropriate $\Psi$ will result in both simplified computations and good accuracy. The $B$-splines (see Schoenberg [8], and later work by de Boor, Schoenberg and others), which are polynomial splines of minimal support, may prove to be useful in the above respects.

Acknowledgements. I wish to thank the referee for suggesting that a necessary and sufficient condition for $\operatorname{det}\left(G^{T} F\right)>0$ for every $F$ (as defined above) could be found by modifying the definition of RSM in [2], and for several helpful references. 
I also thank G. Dahlquist for his encouragement and some illuminating comments.

Department of Computer Sciences, Numerical Analysis

The Royal Institute of Technology

S-100 44 Stockholm 70, Sweden

1. A. C. AITKEN, Determinants and Matrices, Oliver and Boyd, Edinburgh, 1939. MR 1, 35.

2. N. ANDERSON, A Generalized Method of Averages, Report NA 73.05, Royal Institute of Technology, Stockholm, 1973.

3. G. DAHLQUIST \& $\AA$ BJÖRCK, Numerical Methods, Prentice-Hall, Englewood Cliffs, N. J., 1974.

4. G. DAHLQUIST, B. SJÖBERG \& P. SVENSSON, "Comparison of the method of averages with the method of least squares," Math. Comp., v. 22, 1968, pp. 833-846. MR 39 \#1099.

5. S. KARLIN \& W. STUDDEN, Tchebycheff Systems, Interscience, New York, 1966. MR 34 \#4757.

6. G. KOWALEWSKI, Einführung in die Determinantentheorie, 3rd ed., Chelsea, New York, 1948.

7. C. C. MACDUFFEE, Vectors and Matrices, Carus Monograph Ser., no. 7, Math. Assoc. of Amer., 1943. MR 5, 30.

8. I. J. SCHOENBERG, "Contributions to the problem of approximation of equidistant data by analytic functions, Part A," Quart. Appl. Math., v. 4, 1946, pp. 45-99. MR 7, 487. 